\title{
Evaluation of Metabolic Effects of Nusinersen in Patients with Spinal Muscular Atrophy
}

\author{
Lena-Luise Becker ${ }^{1,2,3}$ Claudia Weiss ${ }^{2}$ René Günther ${ }^{4,5}$ Andreas Hermann ${ }^{4,6,7}$ Manuela Theophil ${ }^{8}$ \\ Angela Hübner $^{9}$ Martin Smitka ${ }^{10}$ Maja von der Hagen ${ }^{10}$ Angela M. Kaindl ${ }^{1,2,3}$
}

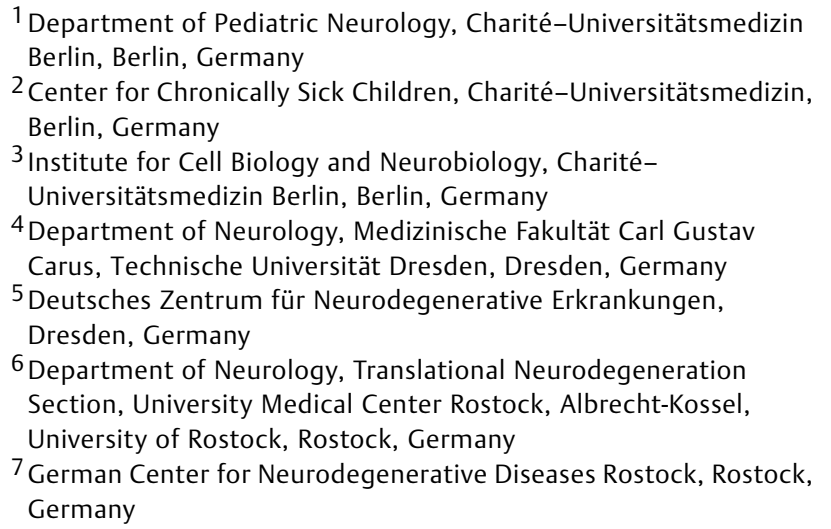
Address for correspondence Angela M. Kaindl, MD, Charité- Universitätsmedizin Berlin, Campus Virchow-Klinikum, Augustenburger Platz 1, 13353 Berlin, Germany
(e-mail: angela.kaindl@charite.de).
${ }^{8}$ Department of Pediatric Neurology, DRK Kliniken Berlin Westend, Berlin, Germany
${ }^{9}$ Department of Pediatrics, Technische Universität Dresden, Medizinische Fakultät Carl Gustav Carus, Dresden, Germany
10 Department of Pediatric Neurology, Technische Universität Dresden, Medizinische Fakultät Carl Gustav Carus, Dresden, Germany

J Pediatr Neurol 2022;20:252-257.

\section{Abstract \\ Keywords \\ - spinal muscular atrophy \\ - nusinersen \\ - endocrinological data \\ - anthropometric data \\ - side effects \\ - weight}

Nusinersen is the first disease-modifying therapy for spinal muscular atrophy (SMA), but there are few data on potential long-term endocrinological and metabolic systemic effects of this novel treatment as well as metabolic alterations in SMA itself. In this retrospective and multicentric study, we analyzed anthropometric, endocrinological, and motor function data of 81 pediatric and adult patients with SMA1 to 3 undergoing treatment with nusinersen. In 39 patients (51\%), we observed a slight increase in body mass index (BMI) centiles under treatment with nusinersen, especially in patients with SMA2 and in pediatric patients between 3.1 and 12 years. A correlation to the SMN2 copy number or motor function was not found. Additionally, length centiles decreased significantly under treatment. The results of longitudinal endocrinological assessments were interpreted as not clinically significant in most patients; in three patients, the signs of an altered glucose metabolism were present. Our study indicates a putative effect of treatment with nusinersen on BMI, which might be due to a conjoint effect of weight gain and reduction of height velocity, without evidence of correlation to increased muscle function. Further studies need to address specific effects of targeted therapies such as nusinersen or onasemnogene abeparvovec on body composition including fat and muscle mass. received

December 17, 2020

accepted after revision

May 2, 2021

published online

July 3,2021
DOI https://doi.org/ 10.1055/s-0041-1731395. ISSN $1304-2580$

\footnotetext{
(C) 2021. The Author(s).

This is an open access article published by Thieme under the terms of the Creative Commons Attribution-NonDerivative-NonCommercial-License, permitting copying and reproduction so long as the original work is given appropriate credit. Contents may not be used for commercial purposes, or adapted, remixed, transformed or built upon. (https://creativecommons.org/ licenses/by-nc-nd/4.0/) Georg Thieme Verlag KG, Rüdigerstraße 14, 70469 Stuttgart, Germany
} 


\section{Introduction}

Spinal muscular atrophy (SMA) is a neuromuscular disease caused by biallelic deletions or mutations in the survival motor neuron 1 gene (SMN1), leading to progressive muscle atrophy and weakness. ${ }^{1}$ SMA is categorized into four subtypes (SMA1-4), depending on onset and motor milestones reached. ${ }^{2}$ Disease variability is attributed partly by the copy numbers of SMN2, a homologous gene of SMN1 that produces mainly a nonfunctional protein due to aberrant splicing. ${ }^{1}$ SMA is primarily a lower motoneuron disease despite the ubiquitous expression of $S M N 1^{3}$, and recent evidence suggests a multisystem involvement. ${ }^{3,4}$ For example, the neuronal degradation of hypothalamic cells and/or an increase in fat content could cause the highly prevalent hyperleptinemia identified in SMA. ${ }^{4}$

The first disease-modifying therapy, nusinersen, is an antisense oligonucleotide that increases SMN2 expression by blocking the aberrant intron silence sequence in the precursor mRNA, thereby promoting the functional protein. ${ }^{1,5}$ So far, studies focusing on extra-nervous system effects of this treatment are rare. ${ }^{2}$ However, studies on the natural course of SMA in patients and heterozygous $S M N$-depleted mice $(S M N+/-)$ lacking the SMA-neuromuscular phenotype, suggested metabolic and anthropometric disturbances involving weight loss in SMA1, an increase in fat mass in SMA patients, disturbances in the glucose metabolism of SMA1 patients (reduction in $\beta$ cells, hyperglycemia, hyperinsulinemia) and in an intermediate SMA mouse model (SMN2B/- mice) ${ }^{2,6-8}$

Recently, in our institutions, clinicians observed an increased body mass index (BMI) and appetite in several patients under nusinersen therapy. To investigate the clinical observation, this retrospective, multicentric study aimed to analyze anthropometric data of pediatric and adult SMA patients undergoing nusinersen treatment to elucidate possible metabolic alterations. To differentiate whether an increase in BMI might be due to endocrinological alterations, we also looked at laboratory parameters.

\section{Patients and Methods}

In a retrospective, multicentric study, we analyzed the clinical data of 81 patients with genetically verified SMA1 to 3 undergoing nusinersen treatment at the Departments of Neuropediatrics at Charité, the Technische Universität Dresden (TUD), the DRK Hospital Berlin Westend and the TUD Department of Neurology. Patients received $12 \mathrm{mg}$ of nusinersen as an intrathecal injection as recommended. ${ }^{9}$ SMN2 copy number was assessed by using the multiplex ligationdependent probe amplification in all individuals. ${ }^{10}$ Due to the retrospective nature of the study, not all measurements were available for all patients. Patients were excluded if baseline anthropometric data were not available. The study was approved by the ethics committee of Charite (EA2/061/ 18) and TUD (EK393122012; EK273062019).

Anthropometric data (weight, length, body mass index [BMI]) were evaluated at every injection time point. Weight was measured by using an electronic scale, with children either lying, sitting, or standing depending on their age and abilities. Length was measured with a standardized stadiometer or by a trained physiotherapist with a measuring tape measuring arm span in patients unable to stand. Ageand gender- adapted centiles (\%) and standard deviations (SD, z-score) were calculated by using the reference dataset of the Robert Koch Institute (www.rki.de). The BMI trend (diff. BMI centiles/SD) was calculated by taking the difference between the BMI/weight/height centile prior treatment and last contact and categorized as lower $(<-0.9 \% / \mathrm{z}$-score $)$, equal ( -0.9 to $0.9 \% / z$-score), or higher $(>0.9 \% / z$-score).

Standardized evaluation of motor function was performed in 68 patients repeatedly with personal trained through the SMArtCARE initiative (Children's Hospital of Philadelphia Infant Test of Neuromuscular Disorders, CHOP [INTEND]; Hammersmith Functional Motor Scale-Expanded, [HFSME]; Revised Upper Limb Module [RULM]). In further 13 patients, no baseline testing was conducted prior to the first treatment. Motor function trend was categorized by using test result points assessed prior to treatment and at last contact: worse (CHOP INTEND $\leq-4$; HFSME $\leq-3$; RULM $\leq-3$ ) and equal (CHOP INTEND: -3 to +3 points; HFSME: -2 to +2 points; RULM: -2 to +2 points); and better (CHOP INTEND $\geq$ $4, \operatorname{HFSME} \geq 3, \operatorname{RULM} \geq 3)^{5,11}$ If we conducted more than one of the above-mentioned tests for a patient, we included the test with the highest improvement.

Endocrinological assessment included fasting blood-glucose level (BG), HbA1c, thyroid-stimulating hormone (TSH), free/total triiodothyronine (fT3/T3), free thyroxine (fT4), insulin-like growth factor-1 (IGF-I), insulin growth factorbinding protein 3 (IGFB3), cortisol, and prolactin. Standardized tests were conducted including age- and sex-related ranges (Berlin: www.laborberlin.com; Dresden: https:// www.uniklinikum- dresden.de/de/das-klinikum/klinikenpolikliniken-institute/ikl); in 20 patients, no laboratory assessment was conducted. Results were evaluated by a pediatric endocrinology specialist.

\section{Statistics}

Statistical analysis was performed with IBM SPSS Statistics version 25.0 (IBM Corp., New York City, United States). Shapiro-Wilk test was used to test for normality. Chi-square test was used to correlate SMA type to age at first dose. The paired Wilcoxon test was used to correlate BMI centiles/SD, weight centiles/SD, and length centiles/SD at first and last contact. Diff. BMI centiles were correlated to gender by using the Mann-Whitney test and to age at first dose, number of doses, SMN2 copy number, and motor function using the Kruskal-Wallis test. Graphs were created by using GraphPad Prism 6 (GraphPad Software; La Jolla, California, United States).

\section{Results}

In our study, 81 pediatric and adult patients ( $49.4 \%$ males and 50.6\%females) with SMA1 (19.7\%), SMA2 (45.7\%), and SMA3 (34.6\%) and a mean age at first dose of 14.1 years 
Table 1 Demographic and clinical characteristics of the cohort

\begin{tabular}{|c|c|c|c|c|c|c|c|c|}
\hline \multicolumn{2}{|l|}{ Category } & \multicolumn{7}{|c|}{ No. $(n \%)$} \\
\hline \multirow[t]{2}{*}{ Sex } & Male & \multicolumn{7}{|c|}{$40(49.4)$} \\
\hline & Female & \multicolumn{7}{|c|}{$41(50.6)$} \\
\hline \multirow{2}{*}{$\begin{array}{l}\text { Age at first } \\
\text { dose }\end{array}$} & Mean & \multicolumn{7}{|c|}{$14.1 \mathrm{y}$} \\
\hline & Min.-max. & \multicolumn{7}{|c|}{$0.4-56$ y } \\
\hline \multicolumn{2}{|c|}{ SMN2 copy number } & 2 & 3 & 4 & 5 & 6 & Unk. & Total, No. $(n \%)$ \\
\hline \multicolumn{2}{|l|}{ SMA type 1} & 9 & 6 & 1 & 0 & 0 & 0 & $16(19.7)$ \\
\hline \multicolumn{2}{|l|}{ SMA type 2} & 4 & 17 & 4 & 3 & 0 & 9 & $37(45.7)$ \\
\hline \multicolumn{2}{|l|}{ SMA type 3} & 4 & 6 & 9 & 3 & 1 & 5 & $28(34.6)$ \\
\hline \multirow{2}{*}{$\begin{array}{l}\text { Observation } \\
\text { period }\end{array}$} & Mean & \multicolumn{7}{|c|}{$11.4 \mathrm{mo}$} \\
\hline & Min.-max. & \multicolumn{7}{|c|}{$5-25 \mathrm{mo}$} \\
\hline
\end{tabular}

Abbreviations: Min., minimum; max., maximum; no., number; SMA, spinal muscular atrophy; SMN, survival motor neuron gene; unk., unknown.

(range $=0.4-56$ years) were observed over a median period of 11.4 months (range $=5-25$ months; - Table 1 ). SMN2 copy number frequency is shown in - Table 1, individual longitudinal BMI/weight/length values in - Supplementary Fig. S1 (available in the online version) and statistical analyses using the age- and gender-adapted SD instead of centiles are shown in -Supplementary Fig. $\mathbf{S 2}$ (available in the online version).

We observed a significant increase in the age- and genderadapted BMI centiles of patients undergoing nusinersen treatment when analyzing pediatric patients only $(p=0.008)$ and the whole cohort $(p=0.03 ;$ - Fig. 1A-C). In total, the BMI centiles under therapy were higher in 39 (51\%), unchanged in 17 (23\%), and lower in 20 (26\%) patients (-Fig. 1H). Weight centiles of pediatric patients did not significantly increase under treatment ( $p=0.35$; - Fig. 1D, -1E). However, in the $48 \%$ of patients, weight centiles did

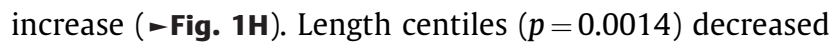
significantly in pediatric patients over the observation period (-Fig. 1F, -1G).
The BMI centiles increased significantly in SMA2 $(p=0.02)$ and showed a trend toward an increase in SMA3 $(p=0.06)$, but did not increase in SMA1 patients $(p=0.99)$ (-Fig. 1I-K). We observed the expected correlation between SMA type and age at first dose $(p<0.0001 ;-$ Fig. $1 \mathbf{M})$. Furthermore, the BMI centiles showed a trend toward a correlation with the age at first dose $(p=0.09)$, most significantly between 3.1 and 12 years, that is, the one with most SMA2 patients (-Fig. 1L). In children in this age group, the BMI centiles significantly increased ( $p=0.0004)$ and also weight centiles showed a trend toward an increase $(p=0.08,-$ Fig. $1 \mathbf{N}$, -10). The diff. BMI centiles correlated with the number of doses received $(p=0.007)$, but not with sex $(p=0.3)$, SMN2 copy number $(p=0.75)$ or motor function in the whole cohort ( $p=0.78$ ) or the subcohort of 3.1 to 12 years old, the subcohort with the highest BMI gain ( $p=0.6$; - Fig. 1P-T).

Longitudinal endocrinological parameters were available from 16 to 61 patients, depending on the analyzed laboratory parameter (-Table 2). Increased HbA1c and BG levels were

Table 2 Overview over pathological laboratory results

\begin{tabular}{|c|c|c|c|c|c|c|}
\hline \multirow{3}{*}{$\begin{array}{l}\text { Laboratory } \\
\text { parameter }\end{array}$} & \multirow{3}{*}{$\begin{array}{l}\text { Total no. } \\
\text { of patients } \\
\text { tested }\end{array}$} & \multirow{3}{*}{$\begin{array}{l}\text { No. of patients } \\
\text { with pathological } \\
\text { laboratory results }\end{array}$} & \multicolumn{4}{|c|}{ No. of measurements of all patients } \\
\hline & & & \multirow[t]{2}{*}{ Total } & \multicolumn{3}{|c|}{ Not within normal range } \\
\hline & & & & Total & Elevated & Decreased \\
\hline BG & 41 & $5 / 41(12.2 \%)$ & 72 & 6 & 6 & 0 \\
\hline HbA1c & 45 & $3 / 45(6.7 \%)$ & 113 & 5 & 5 & 0 \\
\hline TSH & 60 & $2 / 60(3.3 \%)$ & 246 & 12 & 6 & 6 \\
\hline T3/fT3 & 60 & $2 / 60(3.3 \%)$ & 200 & 2 & 2 & 0 \\
\hline fT4 & 61 & $18 / 61(29.5 \%)$ & 203 & 24 & 23 & 1 \\
\hline IGF-I & 61 & $8 / 61(13.1 \%)$ & 194 & 12 & 10 & 2 \\
\hline IGFB3 & 61 & $4 / 61(6.6 \%)$ & 196 & 7 & 1 & 6 \\
\hline cortisol & 60 & $18 / 60(30 \%)$ & 195 & 27 & 7 & 20 \\
\hline prolactin & 16 & 4/16 (25\%) & 71 & 6 & 6 & 0 \\
\hline
\end{tabular}

Abbreviations: BG, fasting blood glucose level; fT3/T3, free and total triiodothyronine; fT4, free thyroxine; IGFB3, insulin-like growth factor-binding protein 3; IGF-I, insulin-like growth factor 1; no., number; TSH, thyroid- stimulating hormone. 
Metabolic Data in Patients with SMA Becker et al. 255
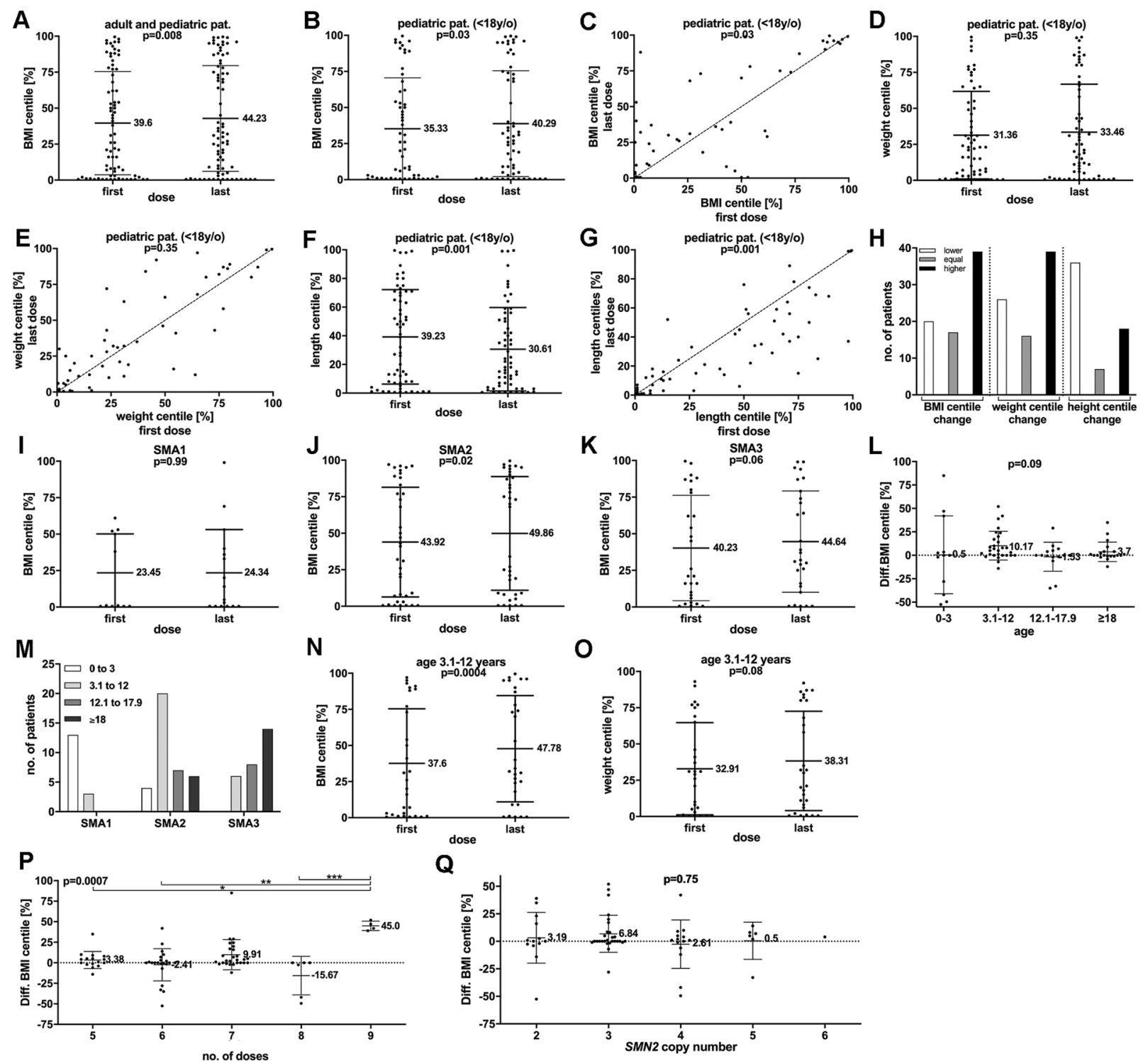

Q
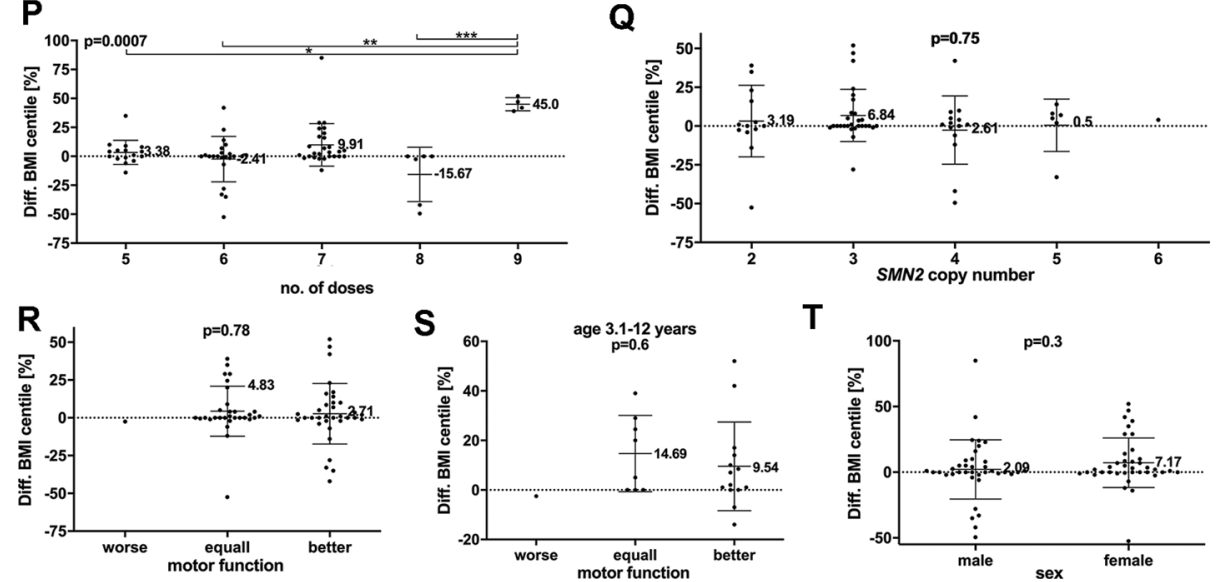

$\mathbf{T}$

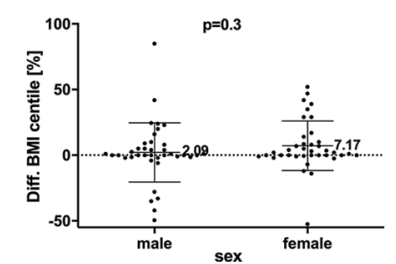

Fig. $1 \mathrm{BMI}$ and weight development of patients with SMA1 to 3 undergoing nusinersen treatment. (A) The BMI centiles of adult and pediatric patients increased under treatment with nusinersen ( $n=76$; Wilcoxon's test; $p=0.008$ ), and also in (B) patients $<18$ years increased under treatment with nusinersen $(n=56$; Wilcoxon's test; $p=0.03$ ). (C) The BMI centiles of patients $<18$ years prior to treatment are above the line of identity $(y=1 \times)$, which shows the high proportion of patient in which the BMI centiles increased. (D) Weight centiles of patients $<18$ years were not significantly increased when comparing first and last injection point (Wilcoxon's test; $p=0.347$ ). (E) The weight centiles of patients $<18$ years prior to treatment are only above the line of identity $(y=1 \times)$ in some patients. $(F)$ Length centiles (Wilcoxon's test; $p=0.0014)$ of patients $<18$ years were significantly decreased when comparing first and last injection time point. (G) The length centiles of patients $<18$ years prior to treatment are below the line of identity $(y=1 \times)$, representing the decrease in length centiles seen within the observation period. (H) BMI centiles were lower in 20 patients (26\%), equal in 17 patients (23\%) and higher in 39 patients (51\%), weight centiles were lower in 26 patients (32\%), equal in 16 patients (20\%) and higher in 39 patients (48\%), and height centiles were lower in 36 patients (44\%), equal in 7 patients (9\%) and higher in 18 patients (22\%) when comparing measurements at first and last conducted injection point. (I) BMI centiles were not higher in SMA1 when comparing first and last injection point (Wilcoxon's test; $p=0.99$ ); however, in (J) SMA2 (Wilcoxon's test; $p=0.02$ ), they increased significantly, but not in (K) SMA3 (Wilcoxon's test; $p=0.06$ ). (L) Diff. BMI centiles trend did not correlate with age of patients at first dose (Kruskal-Wallis test; $p=0.09$ ). (M) SMA type did correlate with age of patients at first dose (Chi-quadrat test; $p<0.001$ ). (N) BMI centiles increased significantly in the subgroup of patients between 3.1 and 12 years (Wilcoxon's test; $p=0.0004$ ), but (O) weight centiles merely showed a trend toward an increase (Wilcoxon's test; $p=0.08$ ). (P) Diff. BMl centiles trend did correlate with the number of doses given (Kruskal-Wallis test, $p=0.0007 ;{ }^{*} p<0.05$; $\left.{ }^{* *} p<0.01 ;{ }^{* * *} p<0.001\right)$, but not with (Q) SMN2 copy number ( $n=61$; Kruskal-Wallis test; $\left.p=0.75\right)$, (R) motor function changes ( $n=64$; Wilcoxon's test; $p=0.78$ ) of the whole cohort or $(S)$ in children between 3.1 and 12 years of age $(n=22$; Wilcoxon's test; $p=0.6)$ or (T) sex (Wilcoxon's test; $p=0.3$ ). BMI, body mass index; Diff. BMI centiles, difference between BMI centiles prior to and under treatment; SMA, spinal muscular atrophy; SMN, survival motor neuron gene. 
detected in two adult patients, but further analysis was impossible due to loss of follow-up. fT4 values were not within normal range in 18/61 (29.5\%) patients but were interpreted as clinically insignificant due to concurrently normal TSH/(f)T3 values. Cortisol values were not within normal range in $18 / 60$ (30\%) patients. However, the values were only mildly elevated ( $<800 \mathrm{nmol} / \mathrm{L} ; n=5$ ) or decreased ( $>50 \mathrm{nmol} / \mathrm{L} ; n=10$ ) in 15 patients once during the observation period. In two patients, we detected mildly decreased cortisol levels several times (four times: $n=2$; two times: $n=1$ ), and in one patient, cortisol levels were mildly elevated twice. None of the patients had clinical signs of hypo- or hypercortisolism. The mildly decreased cortisol values could also be due to the blood not been drawn between 8 and 9 A.M. reflecting the physiologically lower levels during the day. Therefore, we interpreted all measurements as being normal or slightly abnormal without clinical relevance.

\section{Discussion}

The aim of the study was to investigate the clinical observation of an increased BMI in SMA patients undergoing nusinersen treatment and differentiate between various causes of this finding, for example, increase of weight/muscle mass, decrease of length growth velocity, or endocrinological causes. We observed an increase in the BMI centiles in half of the patients, especially in those with SMA2 and between 3.1 and 12 years of age. To evaluate an association between an increased muscle bulk and weight gain, we correlated BMI and motor function, but did not detect a correlation, indicating an alternative effect.

In the natural course of SMA, SMA1 patients are at higher risk of becoming underweight and SMA2 overweight ${ }^{8,12}$ (-Supplementary Table S1 [available in the online version]). Additionally, previous studies identified that 56\% of SMA patients are $<50$ th BMI centile, which is in line with our cohort where $55.6 \%$ of patients were $<50$ th BMI centile prior to treatment ${ }^{12}$ and the mean values of the $\mathrm{BMI} /$ weight centiles were $<50$ th centile in all SMA types, especially in SMA1. The BMI centiles of patients with SMA1 were stable over the observation period in contrast to previous findings of these patients becoming more underweight over time, ${ }^{8}$ indicating a potential beneficial effect of nusinersen on weight in these children. This is important because failure to thrive is an important clinical sign for disease progression. Therefore, this topic needs to be addressed throughout the disease course. ${ }^{13}$ Another study identified an increase in mean weight centiles under nusinersen treatment in infantile-onset SMA by $0.1 \%$ and in the control group by $20.7 \%{ }^{2}$ Weight $(\mathrm{kg})$ in later-onset SMA patients treated with nusinersen compared with control patients did not differ. However, no centiles were reported in these patients. ${ }^{2}$ This is in contrast to our findings, since we observed a BMI increase, especially in later-onset SMA2. Because we do not have nontreated controls, the increase in BMI centiles in patients with SMA2 could also be due to the natural course of SMA itself like reported in previous studies that identified a high proportion of overweight in patients with SMA2. ${ }^{2,12}$
Due to the high prevalence of increased fat mass in SMA ${ }^{6,8,14}$ and the missing data of body composition, further studies need to analyze the effect of nusinersen on body composition including fat and muscle mass. Further, we did not evaluate confounding factors such as nutritional supplementation, high-caloric diet, and dysphagia in our study, which could lead to weight loss/gain in effected individuals.

Recent studies by Darras et al in infants observed a decrease of $16.1 \%$ in mean length centiles in nusinersentreated patients and an increase by $18.6 \%$ in the control group supporting our findings of a significant decrease in length centiles in children during treatment. ${ }^{2}$ However, this could also be due to the natural course in SMA with agerelated progressing of contractures and scoliosis. ${ }^{2}$ Furthermore, laboratory data did not indicate hypothyroidism, hypercortisolism, or growth hormone deficiency as potential causes for the decreased height velocity or weight gain. Therefore, BMI increase could be due to a conjoint effect of a decrease in height velocity and mild weight gain.

In two adult patients, we detected high BG and HbA1c levels and in the cohort was one juvenile patient with type 1 diabetes prior to treatment, indicating a diabetic metabolic state favoring weight gain. In line with our findings in humans, $S M N 2 B /-$ mice were reported to have high glucagon and normal insulin levels. ${ }^{6}$ In addition, SMA1 patients and $S M N 2 B /-$ mice had a reduced number of insulin-producing $\beta$-cells, indicating metabolic dyshomeostasis. ${ }^{6}$ Heterozygous $S M N+/-$ mice without neuromuscular phenotype were heavier, displayed hyperglycemia, hyperinsulinemia, and increased hepatic glucagon sensitization, suggesting an influence of SMN independent of motor function. ${ }^{7}$ Recent studies suggest that patients with SMA may have an increased risk to develop hypoglycemia during fasting, even $<6$ hours, with patients with a lower BMI being more at risk. ${ }^{15}$ Therefore, current recommendations advice against prolonged fasting episode in patients with SMA. ${ }^{16}$ Additionally, earlier studies identified an increase in life expectancy in SMN $\triangle 7$ SMA mice if a maternal diet with higher fat content (PicoLab20) was used. ${ }^{17}$ In contrast to these studies, we did not detect low BG in our patients. Further studies in larger cohorts and after gene (-modifying) therapies like onasemnogene abeparvovec are needed to analyze a potential beneficial effect of these treatments on BG in SMA patients to provide caretakers with more previse recommendations.

These laboratory signs of an altered glucose metabolism as well as a tendency to becoming overweight in SMA may be highly relevant in gene-therapy strategies, potentially implying the long-term application of steroids as concomitant medication. In line with the standard of care recommendations for SMA, we therefore recommend monitoring possible glucose metabolism defects as well as BMI closely. ${ }^{18}$

Earlier studies in SMA patients indicate that SMN depletion results in loss of anterior horn cells but also in chromatolysis of the thalamus and other brain areas ${ }^{19}$ and involvement of multiple organ systems. ${ }^{20}$ Given the role of the paraventricular thalamus in appetite motivation, ${ }^{21}$ nusinersen treatment could impact this system, thereby increasing appetite. ${ }^{4,20}$ 
To conclude, novel treatments for SMA resulting in an increase in patient survival have changed our focus to the long-term outcome, for example, metabolic effects. To understand systemic effects of drugs increasing the amount of SMN in SMA patients, a detailed analysis of the expression of SMN during development in different tissues could enlighten other organ systems that will be affected by these therapies.

\section{Authors' Contributions}

M.S. received compensations for presentations and training activities for Biogen. M.V.D.H. and C.W. received compensations for presentations and taking part in advisory boards from Biogen, Avexis and Sarepta. R.G. has received compensations for presentations/advisory boards from Biogen, and A.M.K. received compensations for taking part in advisory boards from Avexis and GW Pharma. A.H. has received honoraria for presentations/advisory boards from Desitin and Biogen, royalties from Elsevier Press and serves as an editorial board member of BMC neurology.

\section{Funding}

The study was funded by Charité - Universitätsmedizin Berlin and the German Research Foundation (DFG, SFB1315, FOR3004).

\section{Conflict of Interest}

None declared.

\section{Acknowledgments}

The authors thank the patients, their families, and staff who have helped to monitor our patients. They also thank Christoph Hübner for developing the study with us. They thank the SMArtCARE initiative for providing standardized monitoring protocols.

\section{References}

1 Kolb SJ, Kissel JT. Spinal muscular atrophy. Neurol Clin 2015;33 (04):831-846

2 Darras BT, Farrar MA, Mercuri E, et al. An integrated safety analysis of infants and children with symptomatic spinal muscular atrophy (SMA) treated with nusinersen in seven clinical trials. CNS Drugs 2019;33(09):919-932

3 Hamilton G, Gillingwater TH. Spinal muscular atrophy: going beyond the motor neuron. Trends Mol Med 2013;19(01): 40-50

4 Kölbel H, Hauffa BP, Wudy SA, Bouikidis A, Della Marina A, Schara U. Hyperleptinemia in children with autosomal recessive spinal muscular atrophy type I-III. PLoS One 2017;12(03): $\mathrm{e} 0173144$

5 Mercuri E, Darras BT, Chiriboga CA, et al; CHERISH Study Group. Nusinersen versus sham control in later-onset spinal muscular atrophy. N Engl J Med 2018;378(07):625-635
6 Bowerman M, Swoboda KJ, Michalski JP, et al. Glucose metabolism and pancreatic defects in spinal muscular atrophy. Ann Neurol 2012;72(02):256-268

7 Bowerman M, Michalski JP, Beauvais A, Murray LM, DeRepentigny Y, Kothary R. Defects in pancreatic development and glucose metabolism in SMN-depleted mice independent of canonical spinal muscular atrophy neuromuscular pathology. Hum Mol Genet 2014;23(13):3432-3444

8 Poruk KE, Davis RH, Smart AL, et al. Observational study of caloric and nutrient intake, bone density, and body composition in infants and children with spinal muscular atrophy type I. Neuromuscul Disord 2012;22(11):966-973

9 Pechmann A, Langer T, Wider S, Kirschner J. Single-center experience with intrathecal administration of nusinersen in children with spinal muscular atrophy type 1. Eur J Paediatr Neurol 2018; 22(01):122-127

10 Arkblad EL, Darin N, Berg K, et al. Multiplex ligation-dependent probe amplification improves diagnostics in spinal muscular atrophy. Neuromuscul Disord 2006;16(12):830-838

11 Finkel RS, Mercuri E, Darras BT, et al; ENDEAR Study Group. Nusinersen versus sham control in infantile-onset spinal muscular atrophy. N Engl J Med 2017;377(18):1723-1732

12 Sproule DM, Montes J, Montgomery M, et al. Increased fat mass and high incidence of overweight despite low body mass index in patients with spinal muscular atrophy. Neuromuscul Disord 2009;19(06):391-396

13 Mannaa MM, Kalra M, Wong B, Cohen AP, Amin RS. Survival probabilities of patients with childhood spinal muscle atrophy. J Clin Neuromuscul Dis 2009;10(03):85-89

14 Baranello G, De Amicis R, Arnoldi MT, et al. Evaluation of body composition as a potential biomarker in spinal muscular atrophy. Muscle Nerve 2020;61(04):530-534

15 Berti B, Onesimo R, Leone D, et al. Hypoglycaemia in patients with type 1 SMA: an underdiagnosed problem? Arch Dis Child 2020; 105(07):707

16 Mercuri E, Finkel RS, Muntoni F, et al; SMA Care Group. Diagnosis and management of spinal muscular atrophy: part 1: recommendations for diagnosis, rehabilitation, orthopedic and nutritional care. Neuromuscul Disord 2018;28(02):103-115

17 Butchbach ME, Rose FF Jr, Rhoades S, et al. Effect of diet on the survival and phenotype of a mouse model for spinal muscular atrophy. Biochem Biophys Res Commun 2010;391(01):835-840

18 Finkel RS, Mercuri E, Meyer OH, et al; SMA Care group. Diagnosis and management of spinal muscular atrophy: part 2: pulmonary and acute care; medications, supplements and immunizations; other organ systems; and ethics. Neuromuscul Disord 2018;28 (03):197-207

19 Harding BN, Kariya S, Monani UR, et al. Spectrum of neuropathophysiology in spinal muscular atrophy type I. J Neuropathol Exp Neurol 2015;74(01):15-24

20 Lipnick SL, Agniel DM, Aggarwal R, et al. Systemic nature of spinal muscular atrophy revealed by studying insurance claims. PLoS One 2019;14(03):e0213680

21 Millan EZ, Ong Z, McNally GP. Paraventricular thalamus: Gateway to feeding, appetitive motivation, and drug addiction. Prog Brain Res 2017;235:113-137

22 Bertoli S, De Amicis R, Mastella C, et al. Spinal muscular atrophy, types I and II: what are the differences in body composition and resting energy expenditure? Clin Nutr 2017;36(06):1674-1680 